\title{
ON THE RESOLUTION OF CERTAIN GRADED ALGEBRAS
}

\author{
M. P. CAVALIERE, M. E. ROSSI AND G. VALLA
}

\begin{abstract}
Let $A=R / I$ be a graded algebra over the polynomial ring $R=$ $k\left[X_{0}, \ldots, X_{n}\right]$. Some properties of the numerical invariants in a minimal free resolution of $A$ are discussed in the case $A$ is a "Short Graded Algebra". When $A$ is the homogeneous coordinate ring of a set of points in generic position in the projective space, several result are obtained on the line traced by some conjectures proposed by Green and Lazarsfeld in [GL] and Lorenzini in [L1]
\end{abstract}

\section{INTRODUCTION}

If $X \subset \mathbf{P}_{k}^{n}=\mathbf{P}^{n}$ is a projective variety, its homogeneous coordinate ring has a minimal free resolution whose numerical invariants have been the objects of several recent investigations and conjectures (see for example [G, GL, EK, EK1, HSV, L3, BG, TV, CRV2]).

Most of this recent work deals with the linear part of the resolution and in fact it explains in many cases its geometric significance.

In this paper we consider a graded algebra $A:=R / I$ where $R$ is the polynomial ring $R:=k\left[X_{0}, \ldots, X_{n}\right]$ over the algebraically closed field $k$. The graded $R$-module $A$ has a minimal free resolution

$$
\mathbf{E}: 0 \rightarrow E_{h} \rightarrow \cdots \rightarrow E_{1} \rightarrow R \rightarrow A \rightarrow 0
$$

where $h=h d_{R}(A)$ and $E_{p}=\bigoplus_{j=1}^{\beta_{p}} R\left(-d_{p j}\right)$.

If we let $t$ be the initial degree of the homogeneous ideal $I$, we get $d_{p j} \geq$ $p+t-1$ for every $p$ and $j$ and then we let $a_{p}$ be the multiplicity of the minimal shift $p+t-1$ in $E_{p}$.

The resolution $\mathbf{E}$ is said to be linear if $\beta_{p}=a_{p}$ for every $p=1, \ldots, h$. This means that $\mathbf{E}$ is as simple as possible, in the sense that the maps between the $E_{i}$ 's are given by matrices of linear forms.

In [GL] Green and Lazarsfeld generalize this notion by saying that the resolution $\mathbf{E}$ has property $\left(N_{i}\right)$ if $\beta_{p}=a_{p}$ for every $1 \leq p \leq i$. They studied this condition in the case of a finite set of points or an algebraic curve extending and clarifying classical results on generation by quadrics.

Received by the editors February 18, 1991.

1991 Mathematics Subject Classification. Primary 14M05, 13 D99.

Key words and phrases. Resolutions of graded algebras, Koszul complex, points in generic position, minimal resolution conjecture, Green and Lazarsfeld conjecture.

The first two authors were partially supported by M.P.I. (Italy). The third author thanks the MaxPlanck-Institut für Mathematik in Bonn for hospitality and financial support during the preparation of this paper. 
In particular they proved that if $X$ is a set of $2 n+1-i$ points in general position in $\mathbf{P}^{n}(1 \leq i \leq n)$, i.e. with no $n+1$ of them lying on an hyperplane, then $X$ satisfies property $\left(N_{i}\right)$ (see [GL, Theorem 1]).

If $X$ consists of $2 n+1-i$ points, distinct but not necessarily in general position, then $\left(N_{i}\right)$ may fail for $X$ but Green and Lazarsfeld propose the following

Green-Lazarsfeld Conjecture (GLC for short). Let $X$ be a set of $2 n+1-i$ points in $\mathbf{P}^{n}$ with $1 \leq i \leq n$. If $X$ fails to satisfy $\left(N_{i}\right)$ then there is an integer $k<n$ and a subset $Y \subseteq X$ consisting of at least $2 k+2-i$ points such that

(a) $Y$ is contained in a linear subspace $\mathbf{P}^{k} \subset \mathbf{P}^{n}$.

(b) $\left(N_{i}\right)$ fails for $Y$ in $\mathbf{P}^{k}$.

It is easy to see that $G L C$ holds if $s \leq n+2$. In [EK] Eisenbud and Koh proved $G L C$ for six points in $\mathbf{P}^{3}$ and eight points in $\mathbf{P}^{4}$.

A weaker version of this conjecture is the following.

(glc) Let $X$ be a set of $2 n+1-i$ points in $\mathbf{P}^{n}$ with $1 \leq i \leq n$. If for every $k=i, \ldots, n-1$, no $2 k+2-i$ points lie on a linear subspace $\mathbf{P}^{k}$ of $\mathbf{P}^{n}$, then $X$ satisfies property $\left(N_{i}\right)$.

We say that the points of $X$ are in $G L$ position if $X$ verifies the assumptions of the above statement.

One of the main results of this paper is an extension of the result of Green and Lazarsfeld on the way towards a proof of the (glc). For a set $X$ of $\left(\begin{array}{c}n+t-1 \\ n\end{array}\right)+n-i$ distinct points in $\mathbf{P}^{n}$, we introduce the notion of $g l$ position and prove that if the points of $X$ are in $g l$ position then $X$ satisfies $\left(N_{i}\right)$ (see Theorem 2.5). For $t=2$ general position implies $g l$ position and $g l$ position implies $G L$ position, thus proving that our result is on the midway between the theorem and the weaker conjecture by Green and Lazarsfeld.

Since $G L$ position implies $g l$ position for six points in $\mathbf{P}^{3}$ and seven in $\mathbf{P}^{4}$, we get a proof of $g l c$, and in fact of $G L C$, for these numbers of points (see Proposition 2.8).

We remark that in the case of a set $X$ of $s=\left(\begin{array}{c}n+t-1 \\ n\end{array}\right)+n-i$ points in $g l$ position we can prove that the Hilbert function of $X$, or better of the homogeneous coordinate ring $A$ of $X$, is as big as possible. This means that $H_{A}(i):=\operatorname{dim}_{k} A_{i}=\min \left\{s,\left(\begin{array}{c}n+i \\ n\end{array}\right)\right\}$, or, which is the same, that the points of $X$ are in generic position (see [GO]).

This implies that the minimal resolution of $A$ has only two possibly nonzero shifts at every $E_{i}$, namely $i+t-1$ and $i+t$, whose multiplicities we denote by $a_{i}$ and $b_{i}$ respectively.

In [L1] Lorenzini stated a conjecture on the resolution of a general set of points in generic position. With our methods we can rephrase this conjecture in the following way.

Let $X$ be a set of $s$ distinct points in $\mathbf{P}^{n}$ in generic position. Let $t$ be the integer defined by the inequalities

$$
\left(\begin{array}{c}
n+t-1 \\
n
\end{array}\right) \leq s<\left(\begin{array}{c}
n+t \\
t
\end{array}\right)
$$

and let $m$ be the least integer such that

$$
t\left[\left(\begin{array}{c}
n+t \\
t
\end{array}\right)-s\right] \leq m s .
$$


Notice that $m \geq 1$, since $s<\left(\begin{array}{c}n+t \\ t\end{array}\right)$ by the definition of $t$.

Minimal Resolution Conjecture (MRC for short). There exists a not empty open subset of $\left(\mathbf{P}^{n}\right)^{s}$ consisting of sets of points in generic position for which $a_{m+1}=$ $b_{m-1}=0$.

A very natural consequence of our approach proves that the sets of points in generic position with the expected numerical invariants form an open set which of course has to be proved to be not empty.

The starting point of our investigation is a very general result, proved in [CRV2], which provides an explicit way to compute the integers $a_{i+1}$ in the resolution of any graded algebra $A$. Hence in the first section of the paper we use this method to compare the resolution of two graded algebras $A=R / I$ and $B=R / J$ under suitable assumptions.

Then we study some property of the resolution of algebras with maximal Hilbert function, called "Short Graded Algebras" in [CRV1].

The main results for the class of short graded algebras are Proposition 1.6 where an explicit formula connecting the integers $a_{i+1}$ and $b_{i}$ is given, and Proposition 1.7 which provides a sufficient condition for a short graded algebra to verify property $\left(N_{i}\right)$. This last result extends to a considerable extent a theorem proved by Lorenzini in [L3] with a very heavy approach (see [L3, pp. 25-39]).

In $\S 2$ we study the minimal free resolution of those particular short graded algebras which are given by the homogeneous coordinate rings of a finite set of points in generic position.

In this case we can translate the problem of the computation of the integers $a_{i+1}$ into a problem of linear algebra. We produce a matrix $M_{i}$ whose rank is related to the numerical invariants of the resolution by a nice formula proved in Proposition 2.1.

Then we apply this method to prove our main theorem which we have described as a first step towards a proof of the (glc) .

In the last section of the paper we use the previous work to give some sporadic result on the resolution of points in the projective space.

First we are able to compute all the numerical invariants of the resolution of a set of $s=2 n+1-i$ points lying on a rational normal curve in $\mathbf{P}^{n}$ (see Proposition 3.1).

It turns out that if we have $n+3<s \leq 2 n+1$ points on the rational normal curve in $\mathbf{P}^{n}$, they do not satisfy the MRC. For seven points in $\mathbf{P}^{3}$ in general position also the converse holds (see Proposition 3.3).

Finally we describe all the possible resolutions of five points in $\mathbf{P}^{2}$ and six in $\mathbf{P}^{3}$ (see Propositions 3.6 and 3.7).

In particular we prove that we get the same numerical invariants in the resolution of these two different configurations of six points in $\mathbf{P}^{3}$ :

(a) Five points are on a plane, but four are never on a line.

(b) Three points are on a line and the other three points are on another line.

This proves that the minimal resolution cannot distinguish the geometrical configurations of the points. This should be contrasted with a recent theorem of Hoa, Stuckrad and Vogel (see [HSV, Theorem C]) which proves that for $n+2$ points in $\mathbf{P}^{n}$ different configurations correspond to different numerical invariants in the minimal resolution. 


\section{Graded Algebras}

Let $k$ be an algebraically closed field, $R$ the polynomial ring $k\left[X_{0}, \ldots, X_{n}\right]$, $I$ an homogeneous ideal of $R$ with initial degree $t$ and $A$ the graded algebra $R / I$. Let $\mathbf{E}$ be a minimal graded free resolution of $A$ over $R$ :

$$
\mathbf{E}: 0 \rightarrow E_{h} \rightarrow \cdots \rightarrow E_{1} \rightarrow R \rightarrow A \rightarrow 0
$$

where $h=h d_{R}(A)$ and $E_{i}=\bigoplus_{j=1}^{\beta_{i}} R\left(-d_{i j}\right)$. It is well known that $d_{i j} \geq i+t-1$ for every $i$ and $j$. We denote by $a_{i}$ the multiplicity of the shift $i+t-1$ in $E_{i}$.

Since the resolution is minimal we get

$$
a_{i}=\operatorname{dim}_{k}\left(\operatorname{Tor}_{i}^{R}(A, k)_{i+t-1}\right) \text { for every } i=1, \ldots, h .
$$

We can compute $\operatorname{Tor}_{i}^{R}(A, k)$ using a resolution of $k$ which can be obtained by the Koszul complex of $X_{0}, \ldots, X_{n}$. Let $V$ be a fixed $k$-vector space of dimension $n+1$; then the Koszul resolution of $k$ is given by

$$
\begin{aligned}
0 \rightarrow & \bigwedge^{n+1} V \otimes R(-n-1) \stackrel{\delta_{n+1}}{\longrightarrow} \bigwedge^{n} V \otimes R(-n) \\
& \rightarrow \cdots \rightarrow \bigwedge V \otimes R(-1) \stackrel{\delta_{1}}{\longrightarrow} R \rightarrow k \rightarrow 0
\end{aligned}
$$

where the $\delta_{i}$ are the usual Koszul maps.

Since $A_{p}=R_{p}$ for every $p \leq t-1$, tensoring by $A$ and taking graded pieces, one finds that $\operatorname{Tor}_{i}^{R}(A, k)_{i+t-1}$ is the homology of the complex

$$
\bigwedge^{i+1} V \otimes R_{t-2} \longrightarrow \bigwedge^{i} V \otimes R_{t-1} \longrightarrow \bigwedge^{i-1} V \otimes A_{t}
$$

For any $j \geq 1$ we shall denote by $K_{j}$ the kernel of $\delta_{j}$ in degree $t+j$. Thus $K_{j}$ is the kernel of the usual Koszul map $\bigwedge^{j} V \otimes R_{t} \rightarrow \bigwedge^{j-1} V \otimes R_{t+1}$ which is still denoted by $\delta_{j}$.

The following crucial result (proved in [CRV2]) was suggested to us by the Green-Lazarsfeld's paper [GL].

Proposition 1.1. Let $i$ be any integer, $1 \leq i<h$. With the above assumptions and notations, we have

$$
a_{i+1}=\operatorname{dim}_{k}\left[\left(\bigwedge^{i} V \otimes I_{t}\right) \cap K_{i}\right]
$$

Proof. The proof is the same as Proposition 1 of [CRV2].

To compute the dimension of the vector space $K_{i}$ we first need the following easy lemma:

Lemma 1.2. For any $t \geq 1$ and $i, h \geq 0$ we have

$$
\sum_{j=0}^{t-1}(-1)^{t-j+1}\left(\begin{array}{c}
h+j-1 \\
j
\end{array}\right)\left(\begin{array}{c}
h \\
i+t-j
\end{array}\right)=\left(\begin{array}{c}
i+t-1 \\
t-1
\end{array}\right)\left(\begin{array}{c}
h+t-1 \\
i+t
\end{array}\right) .
$$


Proof. We give a proof by induction on $t \geq 1$. If $t=1$ the equality holds. Now

$$
\begin{aligned}
\sum_{j=0}^{t}(-1)^{t-j} & \left(\begin{array}{c}
h+j-1 \\
j
\end{array}\right)\left(\begin{array}{c}
h \\
i+t+1-j
\end{array}\right) \\
= & \sum_{j=0}^{t-1}(-1)^{t-j}\left(\begin{array}{c}
h+j-1 \\
j
\end{array}\right)\left(\begin{array}{c}
h \\
i+1+t-j
\end{array}\right)+\left(\begin{array}{c}
h+t-1 \\
t
\end{array}\right)\left(\begin{array}{c}
h \\
i+1
\end{array}\right)
\end{aligned}
$$

and, by the inductive assumption, this is equal to

$$
-\left(\begin{array}{c}
i+t \\
t-1
\end{array}\right)\left(\begin{array}{c}
h+t-1 \\
i+1+t
\end{array}\right)+\left(\begin{array}{c}
h+t-1 \\
t
\end{array}\right)\left(\begin{array}{c}
h \\
i+1
\end{array}\right)=\left(\begin{array}{c}
i+t \\
t
\end{array}\right)\left(\begin{array}{c}
h+t \\
i+t+1
\end{array}\right)
$$

Now we fix a basis $e_{0}, \ldots, e_{n}$ of $V$. Given a positive integer $r$ we denote by $U(r)$ the set of $r$-tuples $\left\{j_{1}, \ldots, j_{r}\right\}$ such that $0 \leq j_{1}<\cdots<j_{r} \leq n$. Then it is clear that a basis of $\Lambda^{r} V$ is given by the vectors $\left\{\varepsilon_{j}:=e_{j_{1}} \wedge \cdots \wedge e_{j_{r}}\right\}_{j \in U(r)}$. With these notations we prove the following result which is possibly known.

Proposition 1.3. Let $i$ be a positive integer, $1 \leq i \leq n$ and let $K_{i}$ be the kernel of the Koszul map $\bigwedge^{i} V \otimes R_{t} \rightarrow \bigwedge^{i-1} V \otimes R_{t+1}$. Then $\operatorname{dim}_{k} K_{i}=\left(\begin{array}{c}i+t-1 \\ i\end{array}\right)\left(\begin{array}{c}n+t \\ i+t\end{array}\right)$ and $a$ basis of $K_{i}$ is given by the set $S_{i}$ of the vectors $\delta_{i+1}\left(\varepsilon_{j} \otimes T\right)$, where $j \in U(i+1)$ and $T$ runs in the set of monomials of degree $t-1$ in the variables $X_{0}, \ldots, X_{j_{i+1}}$.

Proof. Using the Koszul complex in degree $i+t$ it is clear that the resolution for

is given by

$$
K_{i}=\operatorname{Im}\left(\bigwedge^{i+1} V \otimes R_{t-1} \rightarrow \bigwedge^{i} V \otimes R_{t}\right)
$$

$$
0 \rightarrow \bigwedge^{i+t} V \otimes R_{0} \rightarrow \cdots \rightarrow \bigwedge^{i+1} V \otimes R_{t-1} \rightarrow K_{i} \rightarrow 0
$$

Then

$$
\begin{aligned}
\operatorname{dim}_{k} K_{i} & =\sum_{h=1}^{t}(-1)^{h+1} \operatorname{dim}_{k}\left(\bigwedge^{i+h} V \otimes R_{t-h}\right) \\
& =\sum_{h=1}^{t}(-1)^{h+1}\left(\begin{array}{c}
n+1 \\
i+h
\end{array}\right)\left(\begin{array}{c}
n+t-h \\
t-h
\end{array}\right) \\
& =\sum_{j=0}^{t-1}(-1)^{t-j+1}\left(\begin{array}{c}
n+1 \\
i+t-j
\end{array}\right)\left(\begin{array}{c}
n+j \\
j
\end{array}\right) .
\end{aligned}
$$

If we apply the above lemma we get the first assertion of the proposition.

Now it is clear that $\left\{\delta_{i+1}\left(\varepsilon_{j} \otimes T\right)\right\}$ where $j \in U(i+1)$ and $T$ runs in the set of all monomials of degree $t-1$ in $R$ is a system of generators of $K_{i}$. Let $h$ be the biggest index of the variables in $T$; if $h>j_{i+1}$ and $T=X_{h} P$, we consider the vectors $\xi_{r}=e_{j_{1}} \wedge \cdots \wedge \hat{e}_{j_{r}} \wedge \cdots \wedge e_{j_{i+1}} \wedge e_{h} \otimes X_{j_{r}} P \quad$ for every $r=1, \ldots, i+1$. We remark that $\delta_{i+1}\left(\xi_{r}\right) \in S_{i}$ for every $r$. Now

$$
\delta_{i+2}\left(\varepsilon_{j} \wedge e_{h} \otimes P\right)=(-1)^{i+3}\left(\varepsilon_{j} \otimes X_{h} P\right)+\sum_{r=1}^{i+1}(-1)^{r+1} \xi_{r} .
$$


Hence $\varepsilon_{j} \otimes T-\sum_{r=1}^{i+1}(-1)^{i+r-1} \xi_{r} \in \operatorname{Ker} \delta_{i+1}$ and we have

$$
\delta_{i+1}\left(\varepsilon_{j} \otimes T\right)=\delta_{i+1}\left(\sum_{r=1}^{i+1}(-1)^{r+i-1} \xi_{r}\right)=\sum_{r=1}^{i+1}(-1)^{r+i-1} \delta_{i+1}\left(\xi_{r}\right) .
$$

It follows that the vectors in $S_{i}$ form a system of generators of $K_{i}$. The conclusion follows if we can prove that the cardinality of $S_{i}$ is $\operatorname{dim}_{k} K_{i}$.

Now the number of elements $j \in U(i+1)$ such that $j_{i+1}=k$ is $\left(\begin{array}{l}k \\ i\end{array}\right)$; each of them is multiplied by all the monomials of degree $t-1$ in the variables $X_{0}, \ldots, X_{k}$ which are $\left(\begin{array}{c}k+t-1 \\ k\end{array}\right)$. It follows that the number of elements in $S_{i}$ is

$$
\begin{aligned}
& \sum_{k=i}^{n}\left(\begin{array}{c}
k \\
i
\end{array}\right)\left(\begin{array}{c}
k+t-1 \\
k
\end{array}\right)=\sum_{j=0}^{n-i}\left(\begin{array}{c}
j+i \\
i
\end{array}\right)\left(\begin{array}{c}
i+j+t-1 \\
i+j
\end{array}\right) \\
& \quad=\left(\begin{array}{c}
i+t-1 \\
i
\end{array}\right) \sum_{j=0}^{n-i}\left(\begin{array}{c}
j+i+t-1 \\
j
\end{array}\right)=\left(\begin{array}{c}
i+t-1 \\
i
\end{array}\right)\left(\begin{array}{c}
n+t \\
i+t
\end{array}\right)
\end{aligned}
$$

which is the dimension of $K_{i}$.

If $\Im$ is an ideal of $R$, to avoid confusion, we will sometimes denote by $a_{i}(\Im)$, instead of $a_{i}$, the multiplicity of the minimal shift in each free module of the resolution of $R / \Im$.

We assume $a_{i}(\Im)=0$ if $i=0$ or $i>\operatorname{codim} \Im$.

Let $I$ and $J$ be ideals of $R$ with the same initial degree $t$. We want to compare the resolutions of $R / I$ and $R / J$.

A first trivial result which follows immediately from Proposition 1.1 is the following.

Corollary 1.4. If $I$ and $J$ are ideals of $R$ with the same initial degree $t$ and $J_{t} \subseteq I_{t}$ then $a_{i}(J) \leq a_{i}(I)$ for every integer $i$.

A more subtle result is given by the next proposition where we need some additional assumptions on $I$ and $J$.

Proposition 1.5. Let $I$ and $J$ be ideals of $R$ with the same initial degree $t$. Suppose $I$ is perfect of codimension $h, J_{t} \subseteq I_{t}$ and $\operatorname{depth}(R / J) \geq \operatorname{depth}(R / I)$. Then for every $i=1, \ldots, h$

$$
a_{i}(I) \leq a_{i}(J)+\left(\begin{array}{c}
h \\
i-1
\end{array}\right)\left(\operatorname{dim}_{k} I_{t}-\operatorname{dim}_{k} J_{t}\right) .
$$

Proof. Since $k$ is an infinite field and $I$ is a perfect ideal, we may find linear forms in $R$ which are a maximal regular sequence on $R / I$ and also a regular sequence on $R / J$. Hence we may assume that $I$ and $J$ are ideals of $k\left[X_{1}, \ldots, X_{h}\right]$ of initial degree $\mathrm{t}$ such that $J_{t} \subseteq I_{t}$.

By Proposition 1.1,

$$
a_{i}(I)=\operatorname{dim}_{k}\left[\left(\bigwedge^{i-1} V \otimes I_{t}\right) \cap K_{i-1}\right]
$$

and

$$
a_{i}(J)=\operatorname{dim}_{k}\left[\left(\bigwedge^{i-1} V \otimes J_{t}\right) \cap K_{i-1}\right]
$$

where $V$ is a fixed vector space of dimension $h$. 
We denote by $U$ the $k$-vector space $\Lambda^{i-1} V \otimes I_{t}$ and by $W$ the $k$-vector space $\bigwedge^{i-1} V \otimes J_{t}$; since $W \subseteq U$ it is easy to see that

$$
\operatorname{dim}_{k}\left(U \cap K_{i-1}\right) \leq \operatorname{dim}_{k}\left(W \cap K_{i-1}\right)+\operatorname{dim}_{k} U-\operatorname{dim}_{k} W .
$$

The result follows since $\operatorname{dim}_{k} W=\left(\begin{array}{c}h \\ i-1\end{array}\right) \operatorname{dim}_{k} J_{t}$ and $\operatorname{dim}_{k} U=\left(\begin{array}{c}h \\ i-1\end{array}\right) \operatorname{dim}_{k} I_{t}$.

We shall see later that the inequality given in the above proposition is sharp.

It is clear that Proposition 1.1 becomes more useful if the resolution of $A$ has only two possibly nonzero shifts at every $E_{i}$, namely $i+t-1$ and $i+t$. In fact, in this case, if we know the multiplicities of the minimal shifts, we get all the numerical invariants of the resolution.

We are in this situation if $A$ is a "Short Graded Algebra" (see [CRV1]), that is a Cohen-Macaulay graded algebra whose Poincare series is given by

$$
P_{A}(z)=\left(\sum_{i=0}^{t-1}\left(\begin{array}{c}
h+i-1 \\
i
\end{array}\right) z^{i}+c z^{t}\right) /(1-z)^{d}
$$

Here $d$ is the Krull dimension of $A, t$ is an integer $\geq 2, h$ is the codimension of $I$ and $c$ is an integer such that $0 \leq c<\left(\begin{array}{c}h+t-1 \\ t\end{array}\right)$.

If we denote by $s$ the multiplicity of $A$, we have $s=\left(\begin{array}{c}h+t-1 \\ h\end{array}\right)+c$.

We remark that the integer $t$ defined by the inequalities

$$
\left(\begin{array}{c}
h+t-1 \\
h
\end{array}\right) \leq s<\left(\begin{array}{c}
h+t \\
t
\end{array}\right)
$$

coincides with the socle degree of $A$ and with the initial degree of $A$. From this it follows that a minimal graded free resolution for $A$ is given by

$$
\begin{aligned}
0 & \rightarrow R(-t-h)^{b_{h}} \oplus R(-t-h+1)^{a_{h}} \rightarrow \cdots \rightarrow R(-t-i)^{b_{i}} \oplus R(-t-i+1)^{a_{i}} \\
& \rightarrow \cdots \rightarrow R(-t-1)^{b_{1}} \oplus R(-t)^{a_{1}} \rightarrow R \rightarrow A \rightarrow 0
\end{aligned}
$$

for some $a_{i}, b_{i} \geq 0$.

By the particular Hilbert function of $A$, we get $a_{1}=\left(\begin{array}{c}h+t-1 \\ t\end{array}\right)-c$ and $b_{h}=c$. A detailed proof of these observations can be found in [L2 and CVR1].

In the following we assume that $A=R / I$ is a short graded algebra.

It is clear that in any graded free resolution there is a relationship among the multiplicities of the same shift. For a short graded algebra this connection between $a_{i+1}$ and $b_{i}$ can be read in the following way.

We assume $b_{0}=0$ and $a_{r}=b_{r}=0$ if $r>h$.

Proposition 1.6. Let $i$ be any integer, $0 \leq i \leq h$. With the above assumptions and notations, we have

$$
a_{i+1}=b_{i}+\left(\begin{array}{c}
i+t-1 \\
i
\end{array}\right)\left(\begin{array}{c}
h+t \\
t+i
\end{array}\right)-s\left(\begin{array}{l}
h \\
i
\end{array}\right) .
$$

Proof. Since $A$ is Cohen-Macaulay, we may assume that $X_{0}, X_{h+1}, \ldots, X_{n}$ is a maximal regular sequence on $R / I$. Hence we may prove the result with $R=k\left[X_{1}, \ldots, X_{h}\right]$ and $A$ an artinian graded $k$-algebra with the given free resolution and Poincaré series $P_{A}(X)=\sum_{k=0}^{t-1}\left(\begin{array}{c}h+k-1 \\ k\end{array}\right) X^{k}+\left(s-\left(\begin{array}{c}h+t-1 \\ h\end{array}\right)\right) X^{t}$.

We consider $\sum_{j \geq 0} e_{j} X^{j}$ where

$$
\left\{\begin{array}{l}
e_{0}=1, \\
e_{1}=\cdots=e_{t-1}=0, \\
e_{j}=(-1)^{j-t+1}\left(a_{j-t+1}-b_{j-t}\right) \quad \text { if } j \geq t .
\end{array}\right.
$$


We claim

$$
P_{A}(X)=\frac{\sum_{j \geq 0} e_{j} X^{j}}{(1-X)^{h}}
$$

or, which is the same,

$$
P_{A}(X)=\left(\sum_{j \geq 0} e_{j} X^{j}\right)\left(\sum_{k \geq 0}\left(\begin{array}{c}
h+k-1 \\
k
\end{array}\right) X^{k}\right)
$$

For every $d \geq 0$ we have

$$
\begin{aligned}
\operatorname{dim} A_{d} & =\operatorname{dim} R_{d}+\sum_{i=1}^{d-t+1}(-1)^{i}\left(a_{i}-b_{i-1}\right) \operatorname{dim} R_{d-t-i+1} \\
& =\operatorname{dim} R_{d}+\sum_{j=t}^{d}(-1)^{j-t+1}\left(a_{j-t+1}-b_{j-t}\right) \operatorname{dim} R_{d-j}
\end{aligned}
$$

Now, if we set for short $c_{k}=\operatorname{dim} R_{k}=\left(\begin{array}{c}h+k-1 \\ k\end{array}\right)$, we obtain

$$
\left(\sum_{j \geq 0} e_{j} X^{j}\right)\left(\sum_{k \geq 0} c_{k} X^{k}\right)=e_{0} c_{0}+\left(e_{0} c_{1}+e_{1} c_{0}\right) X+\cdots+\left(\sum_{j=0}^{d} e_{j} c_{d-j}\right) X^{d}+\cdots
$$

But

$$
\begin{aligned}
\sum_{j=0}^{d} e_{j} c_{d-j} & =e_{0} c_{d}+\sum_{j=t}^{d}(-1)^{j-t+1}\left(a_{j-t+1}-b_{j-t}\right) c_{d-j} \\
& =\operatorname{dim} R_{d}+\sum_{j=t}^{d}(-1)^{j-t+1}\left(a_{j-t+1}-b_{j-t}\right) c_{d-j}=\operatorname{dim} A_{d}
\end{aligned}
$$

\section{It follows that}

$$
\begin{aligned}
\sum_{j \geq 0} e_{j} X^{j} & =P_{A}(X)(1-X)^{h} \\
& =\left(\sum_{k=0}^{t-1}\left(\begin{array}{c}
h+k-1 \\
k
\end{array}\right) X^{k}+\left(s-\left(\begin{array}{c}
h+t-1 \\
h
\end{array}\right)\right) X^{t}\right)\left(\sum_{k=0}^{h}(-1)^{k}\left(\begin{array}{l}
h \\
k
\end{array}\right) X^{k}\right)
\end{aligned}
$$

and then for any $i=0, \ldots, h$ we get

$$
\begin{aligned}
a_{i+1}-b_{i} & =(-1)^{i+1} e_{i+t} \\
& =(-1)^{i+1}\left\{\sum_{j=0}^{t-1}(-1)^{i+t-j}\left(\begin{array}{c}
h+j-1 \\
j
\end{array}\right)\left(\begin{array}{c}
h \\
i+t-j
\end{array}\right)\right. \\
& \left.+(-1)^{i}\left(\begin{array}{c}
h \\
i
\end{array}\right)\left[s-\left(\begin{array}{c}
h+t-1 \\
h
\end{array}\right)\right]\right\} \\
& =\sum_{j=0}^{t-1}(-1)^{t-j+1}\left(\begin{array}{c}
h+j-1 \\
j
\end{array}\right)\left(\begin{array}{c}
h \\
i+t-j
\end{array}\right)+\left(\begin{array}{c}
h \\
i
\end{array}\right)\left(\begin{array}{c}
h+t-1 \\
h
\end{array}\right)-s\left(\begin{array}{c}
h \\
i
\end{array}\right) .
\end{aligned}
$$

Since it is easy to see that $\left(\begin{array}{c}i+t-1 \\ t-1\end{array}\right)\left(\begin{array}{c}h+t-1 \\ i+t\end{array}\right)+\left(\begin{array}{c}h \\ i\end{array}\right)\left(\begin{array}{c}h+t-1 \\ h\end{array}\right)=\left(\begin{array}{c}i+t-1 \\ i\end{array}\right)\left(\begin{array}{c}h+t \\ i+t\end{array}\right)$, the conclusion follows by Lemma 1.2. 
Several authors (see [GL, EK, MNV]) are interested in knowing when the minimal graded free resolution $\mathbf{E}$ of a graded $k$-algebra $A$ is "right linear". More precisely, for a given integer $i \geq 1$, they study under which assumptions $A$ satisfies the following property:

$$
E_{j}=R(-j-t+1)^{a_{j}} \text { for } 1 \leq j \leq i
$$

where $t$ is the initial degree of $A$.

In [GL] it is proved that if $A$ is the homogeneous coordinate ring of a set of $2 n+1-i$ points in linear general position in $\mathbf{P}^{n}$, i.e. with no $n+1$ of them lying on a hyperplane, then $A$ satisfies $\left(N_{i}\right)$.

In [EK and MNV] extensions are given of the above result. In each of these results $A$ is a short graded algebra.

It is clear that, for a short graded algebra $A$, the property $\left(N_{i}\right)$ is equivalent to $b_{i}=0$; in fact, since $A$ is Cohen-Macaulay, if $b_{i}=0$, then $b_{j}=0$ for $1 \leq j \leq i$.

The last result of this section gives a first application of Proposition 1.5. It concerns a useful criterion for a short graded algebra to have a right linear resolution.

Proposition 1.7. Let $I$ and $J$ be ideals of codimension $h$ in $R$ with the same initial degree $t$. Suppose that $J_{t} \subseteq I_{t}$ and $R / I, R / J$ are short graded algebras.

If property $\left(N_{i}\right)$ holds for $R / J$, the same is true for $R / I$.

Proof. Let $p:=\operatorname{dim}_{k} I_{t}-\operatorname{dim}_{k} J_{t}$; since $R / I$ and $R / J$ are short graded algebras, by their particular Hilbert function, we get that $s(J)-s(I)=p$ where $s(I)$ stands for the multiplicity of $R / I$ and the analogous for $s(J)$.

Since $R / J$ satisfies $\left(N_{i}\right)$, by Proposition 1.6 we have $a_{i+1}(J)=\left(\begin{array}{c}i+t-1 \\ i\end{array}\right)\left(\begin{array}{c}h+t \\ t+i\end{array}\right)-$ $s(J)\left(\begin{array}{l}h \\ i\end{array}\right)$; moreover by Proposition 1.5 we have

$$
\begin{aligned}
a_{i+1}(I) & \leq a_{i+1}(J)+\left(\begin{array}{c}
h \\
i
\end{array}\right) p=\left(\begin{array}{c}
i+t-1 \\
i
\end{array}\right)\left(\begin{array}{c}
h+t \\
t+i
\end{array}\right)-(s(J)-p)\left(\begin{array}{c}
h \\
i
\end{array}\right) \\
& =\left(\begin{array}{c}
i+t-1 \\
i
\end{array}\right)\left(\begin{array}{c}
h+t \\
t+i
\end{array}\right)-s(I)\left(\begin{array}{c}
h \\
i
\end{array}\right) .
\end{aligned}
$$

Using again Proposition 1.6, we get the result.

\section{PoInTs IN $\mathbf{P}^{n}$}

Let $X$ be a set of $s \geq n+1$ distinct points in $\mathbf{P}^{n}$, not contained in any hyperplane. We denote by $I$ the defining ideal of $X$ in the polynomial ring $R:=k\left[X_{0}, \ldots, X_{n}\right]$ and by $A$ the homogeneous coordinate ring of $X, A:=$ $R / I$. We assume that $t$ is the initial degree of $I$.

After a suitable changing of coordinates, we may assume that the first $n+1$ points of $X$ are the coordinate points, that is $X=\left\{P_{0}, \ldots, P_{n}, Q_{1}, \ldots\right.$, $\left.Q_{s-n-1}\right\}$, where $P_{0}:=(1,0, \ldots, 0), \ldots, P_{n}:=(0,0, \ldots, 1)$ and $Q_{k}:=$ $\left(u_{k 0}, u_{k 1}, \ldots, u_{k n}\right)$ for $k=1, \ldots, s-n-1$.

We want now to translate the problem of the computation of the integers $a_{i+1}$ into a problem of linear algebra.

By Proposition 1.1 we must compute the dimension of the $k$-vector space $\left(\bigwedge^{i} V \otimes I_{t}\right) \cap K_{i}$. Let $\alpha \in \bigwedge^{i} V \otimes I_{t}$; using the notations introduced after Proposition 1.1, we can write $\alpha=\sum_{p \in U(i)} \varepsilon_{p} \otimes F_{p}$ with $F_{p} \in I_{t}$. This implies that the 
$F_{p}$ 's must vanish on $P_{0}, \ldots, P_{n}$, hence in $F_{p}$ we cannot have monomials of the form $X_{h}^{t}$ for every $h=0, \ldots, n$. Since $\alpha \in K_{i}$, by Proposition $1.2 \alpha$ is also a linear combination of the vectors $\delta_{i+1}\left(\varepsilon_{j} \otimes T\right)$ where $j \in U(i+1)$ and $T$ is a monomial of degree $t-1$ in $X_{0}, \ldots, X_{j_{i+1}}$. If $\alpha=\lambda \delta_{i+1}\left(\varepsilon_{j} \otimes X_{h}^{t-1}\right)+\cdots$ with $h \in j$, we get $\alpha= \pm \lambda\left(e_{j_{1}} \wedge \cdots \wedge \hat{e}_{h} \wedge \cdots \wedge e_{j_{i+1}} \otimes X_{h}^{t}\right)+\cdots$.

It follows from this and the remark on the $F_{p}$ 's that $\lambda$ should be zero.

This means that we can delete from the given basis $S_{i}$ of $K_{i}$ the vectors $\delta_{i+1}\left(\varepsilon_{j} \otimes X_{h}^{t-1}\right)$ with $j \in U(i+1)$ and $h \in j$. It is clear that the number of these vectors is $\left(\begin{array}{c}n+1 \\ i+1\end{array}\right)(i+1)=(n+1)\left(\begin{array}{c}n \\ i\end{array}\right)$ and we are left with $\operatorname{dim}_{k} K_{i}-(n+1)\left(\begin{array}{c}n \\ i\end{array}\right)$ vectors which we are going to describe in a more suitable way.

For every $j \in U(i+1)$ we denote by $W(j)$ the set of $\left(j_{i+1}+1\right)$-tuples of nonnegative integers $h:=\left(h_{0}, \ldots, h_{j_{i+1}}\right)$ with $\sum h_{r}=t-1$ and $h_{r}<t-1$ if $r \in j$.

Moreover if $j \in U(i+1)$ and $h \in W(j)$ we let

$$
T_{h}:=X_{0}^{h_{0}} X_{1}^{h_{1}} \cdots X_{j_{i+1}}^{h_{j_{i+1}}}
$$

With these notations we can write an element $\alpha \in\left(\bigwedge^{i} V \otimes I_{t}\right) \cap K_{i}$ in this way

$$
\alpha=\sum_{j, h} \lambda_{j h} \delta_{i+1}\left(\varepsilon_{j} \otimes T_{h}\right)
$$

where $j \in U(i+1), h \in W(j)$ and $\lambda_{j h} \in k$.

If we rewrite $\alpha=\sum_{p \in U(i)}\left(\varepsilon_{p} \otimes F_{p}\right)$, the $F_{p}$ 's must vanish on the "extra" points $Q_{1}, \ldots, Q_{s-n-1}$.

We get in this way $(s-n-1)\left(\begin{array}{c}n+1 \\ i\end{array}\right)$ linear equations $F_{p}\left(Q_{k}\right)=0$ in the $\lambda_{j h}$ which determine a matrix $M_{i}$ of size $(s-n-1)\left(\begin{array}{c}n+1 \\ i\end{array}\right) \times\left(\operatorname{dim}_{k} K_{i}-(n+1)\left(\begin{array}{c}n \\ i\end{array}\right)\right)$.

We can label the rows of $M_{i}$ by $p k, p \in U(i), k=1, \ldots, s-n-1$ and the columns by $j h, j \in U(i+1), h \in W(j)$. Since we have

$$
\alpha=\sum_{j, h} \lambda_{j h}\left(\sum_{r=1}^{i+1}(-1)^{r+1} e_{j_{1}} \wedge \cdots \wedge \hat{e}_{j_{r}} \wedge \cdots \wedge e_{j_{i+1}} \otimes X_{j_{r}} T_{h}\right)
$$

the matrix $M_{i}$ can be easily described as follows:

$$
M_{i}(p k, j h)= \begin{cases}(-1)^{r+1}\left(X_{j_{r}} T_{h}\left(Q_{k}\right)\right) & \text { if } p=\left\{j_{1}, \ldots, \hat{j}_{r}, \ldots, j_{i+1}\right\} \\ 0 & \text { otherwise }\end{cases}
$$

Now it is clear from this description that we get the following formula for $a_{i+1}$.

Proposition 2.1. If $1 \leq i<n$, with the above notations we have

$$
a_{i+1}+\operatorname{rank}\left(M_{i}\right)=\operatorname{dim}_{k} K_{i}-(n+1)\left(\begin{array}{c}
n \\
i
\end{array}\right)
$$

If $A$ is a short graded algebra this result can be coupled with Proposition 1.6 to get all the numerical invariants of the resolution.

In the case of a set $X$ of distinct points, the homogeneous coordinate ring $A$ of $X$ is a short graded algebra if and only if the Hilbert function of $A$ is as big as possible, that is $H_{A}(t):=\operatorname{dim}_{k} A_{t}=\min \left\{s,\left(\begin{array}{c}n+t \\ n\end{array}\right)\right\}$ or, which is the same, the points of $X$ are in generic position (see [GO]). 
It is possible to prove that almost every set of $s$ points in $\mathbf{P}^{n}$ is in generic position, in the sense that the points in generic position in $\mathbf{P}^{n}$ form a dense open subset of $\left(\mathbf{P}^{n}\right)^{s}$.

Remark 2.2. Let $X$ be a set of $s$ points in generic position in $\mathbf{P}^{n}$ and $t$ the integer defined by the inequalities

$$
\left(\begin{array}{c}
n+t-1 \\
n
\end{array}\right) \leq s<\left(\begin{array}{c}
n+t \\
n
\end{array}\right)
$$

Using Propositions 1.2, 1.6 and 2.1 we have

$$
a_{i+1}=b_{i}+\operatorname{dim}_{k} K_{i}-s\left(\begin{array}{c}
n \\
i
\end{array}\right)=\operatorname{dim}_{k} K_{i}-(n+1)\left(\begin{array}{c}
n \\
i
\end{array}\right)-\operatorname{rank}\left(M_{i}\right) .
$$

Hence we get $\operatorname{rank}\left(M_{i}\right)=(s-n-1)\left(\begin{array}{l}n \\ i\end{array}\right)-b_{i} \leq(s-n-1)\left(\begin{array}{l}n \\ i\end{array}\right)$.

Since the size of $M_{i}$ is $(s-n-1)\left(\begin{array}{c}n+1 \\ i\end{array}\right) \times\left(\operatorname{dim}_{k} K_{i}-(n+1)\left(\begin{array}{l}n \\ i\end{array}\right)\right)$ it follows that the maximum possible value for $\operatorname{rank}\left(M_{i}\right)$ is the integer $\min \left\{\operatorname{dim}_{k} K_{i}-\right.$ $\left.(n+1)\left(\begin{array}{l}n \\ i\end{array}\right),(s-n-1)\left(\begin{array}{l}n \\ i\end{array}\right)\right\}$.

It is natural to guess that in the "generic" case this maximum is achieved.

This leads to the following conjecture:

$$
\begin{aligned}
& \text { if } s\left(\begin{array}{c}
n \\
i
\end{array}\right)<\operatorname{dim}_{k} K_{i}, \quad \text { then } \operatorname{rank}\left(M_{i}\right)=(s-n-1)\left(\begin{array}{c}
n \\
i
\end{array}\right) \\
& \text { or equivalently } b_{i}=0, a_{i+1}=\operatorname{dim}_{k} K_{i}-s\left(\begin{array}{c}
n \\
i
\end{array}\right), \\
& \text { if } s\left(\begin{array}{c}
n \\
i
\end{array}\right) \geq \operatorname{dim}_{k} K_{i}, \quad \text { then } \operatorname{rank}\left(M_{i}\right)=\operatorname{dim}_{k} K_{i}-(n+1)\left(\begin{array}{c}
n \\
i
\end{array}\right) \\
& \quad \text { or equivalently } a_{i+1}=0, b_{i}=s\left(\begin{array}{c}
n \\
i
\end{array}\right)-\operatorname{dim}_{k} K_{i} .
\end{aligned}
$$

It is easy to see that we can rewrite $s\left(\begin{array}{l}n \\ i\end{array}\right) \geq \operatorname{dim}_{k} K_{i}$ as $t\left[\left(\begin{array}{c}n+t \\ t\end{array}\right)-s\right] \leq s i$ and this gives the above formulation for the MRC.

This remark leads in a very natural way to the expected numerical resolution and also proves that the sets of $s$ points having this numerical resolution form an open set of $\left(\mathbf{P}^{n}\right)^{s}$. The main problem is to prove that this set is not empty.

For points in the generic position Corollary 1.4 and Proposition 1.7 immediately give a result obtained with much effort by Lorenzini in [L3].

If $X$ consists of points in the generic position, when it is necessary we denote the integers $a_{i}$ and $b_{i}$ by $a_{i}(X)$ and $b_{i}(X)$ respectively and we say that property $\left(N_{i}\right)$ holds for $X$ if it holds for the homogeneous coordinate ring of $X$.

Theorem 2.3. Let $X$ be a set of $s$ points in generic position, $\left(\begin{array}{c}n+t-1 \\ n\end{array}\right) \leq s<\left(\begin{array}{c}n+t \\ n\end{array}\right)$.

(a) Suppose $s<\left(\begin{array}{c}n+t \\ n\end{array}\right)-1$. If $a_{i}(X)=0$, then $X$ is a subset of a set $Y$ consisting of $s+1$ points in generic position with $a_{i}(Y)=0$.

(b) Suppose $s>\left(\begin{array}{c}n+t-1 \\ n\end{array}\right)$. If property $\left(N_{i}\right)$ holds for $X$, then there is a subset $Y$ of $X$ consisting of $s-1$ points in generic position for which $\left(N_{i}\right)$ holds.

Proof. We denote by $I(X)$ and $I(Y)$ the ideals of $X$ and $Y$ respectively.

(a) It is clear that we may choose a point $P$ such that if $Y=X \cup\{P\}$ we have $I(Y)_{t} \varsubsetneqq I(X)_{t}$. Hence $H_{X}(t)<H_{Y}(t)$ and then $Y$ consists of points in 
generic position. Since $s+1<\left(\begin{array}{c}n+t \\ n\end{array}\right)$, the initial degree of $I(Y)$ is again $t$ and so the conclusion follows by Corollary 1.4 .

(b) It is known that it is always possible to take a point $P$ off of $X$ in such a way that $Y=X \backslash\{P\}$ consists of points in generic position. Since $s-1 \geq\left(\begin{array}{c}n+t-1 \\ n\end{array}\right)$, the initial degree of $I(Y)$ is again $t$ and the conclusion follows by Proposition 1.7 .

We now come to the main result of this section. The main tool is the following lemma which plays a central role in the paper.

Proposition 2.4. Let $X$ be a set of $s$ points in generic position in $\mathbf{P}^{n}$. Let $i$ be a positive integer, $1 \leq i \leq n$; then the following facts are equivalent:

(1) $X$ satisfies $\left(N_{i}\right)$,

(2) $b_{i}=0$

(3) $a_{i+1} \leq\left(\begin{array}{c}i+t-1 \\ i\end{array}\right)\left(\begin{array}{c}n+t \\ i+t\end{array}\right)-s\left(\begin{array}{c}n \\ i\end{array}\right)$,

(4) $\operatorname{rank}\left(M_{i}\right) \geq(s-n-1)\left(\begin{array}{c}n \\ i\end{array}\right)$.

Proof. We have seen that $b_{i}=0$ implies $b_{j}=0$ for every $j \leq i$, hence (1) $\Leftrightarrow(2)$. Now (2) $\Leftrightarrow$ (3) by Proposition 1.6 and (3) $\Leftrightarrow$ (4) by Proposition 2.1.

Theorem 2.5. Let $X \subseteq \mathbf{P}^{n}$ be a set of $s=\left(\begin{array}{c}n+t-1 \\ n\end{array}\right)+n-i$ points, $1 \leq i \leq n$, with the following properties:

(a) there are $n$ points of $X$ spanning an hyperplane which does not contain other points of $X$.

(b) any set of $\left(\begin{array}{c}n+t-1 \\ n\end{array}\right)$ points of $X$ containing all the others $s-n$ points of $X$ is in generic position.

Then

(i) $X$ consists of points in generic position.

(ii) $X$ satisfies $\left(N_{i}\right)$.

Proof. After a suitable changing of coordinates, we may assume that the hyperplane as in (a) is $X_{n}=0$ and $X=\left\{P_{0}, \ldots, P_{n}, Q_{1}, \ldots, Q_{s-n-1}\right\}$ where $P_{0}, \ldots, P_{n}$ are the coordinate points of $\mathbf{P}^{n}$.

(i) Let us consider the set $Y=X \backslash\left\{P_{0}, \ldots, P_{n-i-1}\right\}$ which is a subset of $X$ consisting of $\left(\begin{array}{c}n+t-1 \\ n\end{array}\right)$ points. From (b) the points of $Y$ are in generic position. Denote by $I$ and $J$ the defining ideals of $X$ and $Y$ respectively. Since $I \subseteq J$ the initial degree of $I$ is $t$. Now

$$
\begin{aligned}
H_{X}(t) & =\operatorname{dim}_{k} R_{t}-\operatorname{dim}_{k} I_{t}=H_{Y}(t)+\operatorname{dim}_{k} J_{t}-\operatorname{dim}_{k} I_{t} \\
& =\left(\begin{array}{c}
n+t-1 \\
n
\end{array}\right)+\operatorname{dim}_{k} J_{t}-\operatorname{dim}_{k} I_{t} .
\end{aligned}
$$

Hence the conclusion follows if we can prove that $\operatorname{dim}_{k} J_{t}-\operatorname{dim}_{k} I_{t} \geq n-i$.

Since every point $Q_{j}:=\left(u_{j 0}, \ldots, u_{j n}\right)$ has $u_{j n} \neq 0$, we can find $\lambda \in k$ such that $\lambda \neq 0, \sum_{h=n-i}^{n-1} \frac{u_{j h}}{u_{j_{n}}}$. Hence every point of $Y$ does not lie on the hyperplane $\lambda X_{n}=X_{n-i}+\cdots+X_{n-1}$ and this implies that $F:=X_{n-i}+\cdots+X_{n-1}-\lambda X_{n}$ is a regular element modulo $J$. By the particular Hilbert function of $Y$ we have $J+(F) /(F)=\left(X_{0}, \ldots, X_{n}\right)^{t}+(F) /(F)$, hence $X_{j}^{t} \in J+(F)$ for any $j=0, \ldots, n-i-1$. This implies that for every $j$ we can find suitable $G_{j} \in R_{t-1}$ such that the forms $F_{j}:=X_{j}^{t}+F G_{j}$ are in $J_{t}$. Now it is clear that 
$F_{0}, \ldots, F_{n-i-1}$ are linearly independent and they generate a subspace $W$ of $R_{t}$ such that $W \cap I_{t}=0$. It follows that $\operatorname{dim}_{k} J_{t}-\operatorname{dim}_{k} I_{t} \geq n-i$ as desired.

(ii) Since $X$ consists of points in generic position, by Proposition 2.4 we must prove that $\operatorname{rank}\left(M_{i}\right) \geq(s-n-1)\left(\begin{array}{c}n \\ i\end{array}\right)$. As above we denote by $j h \quad(j \in U(i+1)$ and $h \in W(j))$ the columns and by $p k(p \in U(i), k=1, \ldots, s-n-1)$ the rows of $M_{i}$.

It is clear that if we fix $j \in U(i+1)$ with $j_{i+1}=n$ then $h$ runs in the set $W(j)$ consisting of the exponents of all monomials of degree $t-1$ in $X_{0}, \ldots, X_{n}$ different from $X_{j_{1}}^{t-1}, \ldots, X_{j_{i+1}}^{t-1}$. Hence $W(j)$ has $\left(\begin{array}{c}n+t-1 \\ n\end{array}\right)-(i+$ 1) $=s-n-1$ elements. Thus if we consider the submatrix $M_{i}^{\prime}$ of $M_{i}$ obtained by fixing the columns $j h$ with $j_{i+1}=n$ and the rows $p k$ with $p_{i}<n$, we get a square matrix of size $(s-n-1)\left(\begin{array}{c}n \\ i\end{array}\right)$. We shall prove that $\operatorname{det}\left(M_{i}^{\prime}\right) \neq 0$.

Now it is clear that for a fixed $j \in U(i+1)$ with $j_{i+1}=n$, on the $s-n-1$ columns $j h$ we have possibly nonzero entries $(-1)^{i+1}\left(X_{n} T_{h}\right)\left(Q_{k}\right)$ only on the $s-n-1$ rows $i k$ with $i=\left\{j_{1}, \ldots, j_{i}\right\}$.

Hence $M_{i}^{\prime}$ splits in $\left(\begin{array}{c}n \\ i\end{array}\right)$ blocks of size $(s-n-1)$ whose determinant is $\pm\left(u_{1 n} \cdots u_{(s-n-1) n} d_{j}\right)$ where $d_{j}$ is the determinant of the matrix

$$
A_{j}=\left\{T_{h}\left(Q_{k}\right)\right\}_{h \in W(j)} ; k=1, \ldots, s-n-1 .
$$

Since $Q_{1}, \ldots, Q_{s-n-1}$ do not lie on the hyperplane $X_{n}=0$, we have $u_{j n} \neq 0$ for every $j=1, \ldots, s-n-1$. The conclusion follows if we can prove that $d_{j} \neq 0$ for every $j$.

But if $d_{j}=0$ for some $j \in U(i+1)$, then the square matrix of size $\left(\begin{array}{c}n+t-1 \\ n\end{array}\right)$, whose rows are given by all monomials of degree $t-1$ computed on $Q_{1}, \ldots, Q_{s-n-1}, P_{j_{1}}, \ldots, P_{j_{i+1}}=P_{n}$ respectively, would have the following shape

$$
\left(\begin{array}{cc}
A_{j} & \cdot \\
0 & I_{i+1}
\end{array}\right)
$$

and thus its determinant would be zero. This implies that we can find an hypersurface of degree $t-1$ passing through a set of $\left(\begin{array}{c}n+t-1 \\ n\end{array}\right)$ points of $X$ containing $P_{n}, Q_{1}, \ldots, Q_{s-n-1}:$ a contradiction.

In the following if a set of points satisfies conditions (a) and (b) of the above theorem we say that they are in $g l$ position.

It is clear that if $t=2$, Theorem 2.5 extends Green and Lazarsfeld's result (see [GrL, Theorem 1]). In fact, if we have $2 n+1-i$ points in general position, then no $n+1$ of them lie on a hyperplane, and so they are in $g l$ position.

On the other hand the following result shows that our theorem is a step towards the proof of the Green and Lazarsfeld conjecture.

Proposition 2.6. Let $X \subset \mathbf{P}^{n}$ be a set of $2 n+1-i$ points $(1 \leq i \leq n)$ in $g l$ position. Then the points of $X$ are in GL position.

Proof. Let $P_{1}, \ldots, P_{n}$ be the points generating the hyperplane $\pi$ which does not contain other points of $X$; let $Y:=X \backslash\left\{P_{1}, \ldots, P_{n}\right\}$. Let us assume by contradiction that there exists a set $Z \subseteq X$ consisting of $2 k+2-i$ points on a linear subspace $\mathbf{P}^{k}$ of $\mathbf{P}^{n}$ with $i \leq k \leq n-1$.

Let $p$ be the number of points in $Z \cap\left\{P_{1}, \ldots, P_{n}\right\}$. Then $p \leq k+1$ since $P_{1}, \ldots, P_{n}$ are independent points of $\pi$. If $p=k+1$, the $n+k-i+1$ 
points of $Z \cup\left\{P_{1}, \ldots, P_{n}\right\}$ are on an hyperplane which is necessarily $\pi$. Since $n+k-i+1 \geq n+1$ we get a contradiction.

If $p \leq k$, the points of $Y \cap Z$ are at least $2 k+2-i-k=k+2-i$. If we adjoin to $Z$ the remaining points of $Y$, which are at most $n+1-i-(k+2-i)=$ $n-k-1$, we get a set of $n+k-i+1$ points on an hyperplane. Since $n+k-i+1 \geq n+1$ and this set contains $Y$, this gives a contradiction.

We produce now two examples showing that for a set of $2 n+1-i$ points the inclusions of sets

$$
\begin{aligned}
\text { \{Points in general position }\} & \subseteq \text { PPoints in } g l \text { position }\} \\
& \subseteq \text { Points in } G L \text { position }\}
\end{aligned}
$$

are strict. This proves that our extension of Green and Lazarsfeld's theorem is consistent but does not prove the $G L$ conjecture.

Example 1. Let $X=\left\{P_{0}, P_{1}, P_{2}, P_{3}, Q_{1}, Q_{2}\right\} \subset \mathbf{P}^{3}$ where $P_{0}, P_{1}, P_{2}, P_{3}$ are the coordinate points of $\mathbf{P}^{3}$ and $Q_{1}=(1,1,1,1), Q_{2}=(-1,2,1,1)$. The plane $X_{2}-X_{3}=0$ contains the points $P_{0}, P_{1}, Q_{1}, Q_{2}$, so the points are not in general position. Now the plane $X_{3}=0$ contains only the points $P_{0}, P_{1}, P_{2}$, and the plane $X_{0}+2 X_{1}-3 X_{2}=0$ contains only the points $P_{3}, Q_{1}, Q_{2}$. Hence the points of $X$ are in $g l$ position.

Example 2. Let $X=\left\{P_{0}, \ldots, P_{5}, Q_{1}, Q_{2}\right\} \subset \mathbf{P}^{5}$ where $P_{0}, \ldots, P_{5}$ are the coordinate points of $\mathbf{P}^{5}$ and $Q_{1}:=(1,1,1,1,0,1), Q_{2}:=(1,1,2,2,3,0)$.

It is not difficult to see that seven points of them are not on a $\mathbf{P}^{4}$ and 5 are not on a $\mathbf{P}^{3}$, that is the points are in $G L$ position. We prove now that they are not in $g l$ position .

In fact $X_{4}=0$ is a hyperplane containing $P_{0}, P_{1}, P_{2}, P_{3}, P_{5}, Q_{1}, X_{5}=0$ contains $P_{0}, P_{1}, P_{2}, P_{3}, P_{4}, Q_{2}, X_{0}-X_{1}=0$ contains $P_{2}, P_{3}, P_{4}, P_{5}, Q_{1}, Q_{2}$ and $X_{2}-X_{3}=0$ contains $P_{0}, P_{1}, P_{4}, P_{5}, Q_{1}, Q_{2}$. Now it is easy to see that, however we choose three points of $X$, they lie on one of the above hyperplanes which contain six points of $X$. Thus our set $X$ cannot verify condition (b) in Theorem 2.5 .

However we can prove that the converse of Proposition 2.6 holds if $X$ consists of six points in $\mathbf{P}^{3}$ or seven points in $\mathbf{P}^{4}$. Hence, in these cases, Theorem 2.5 proves Green and Lazarsfeld's conjecture. In fact in [CRV3] we prove that for $n+3$ points spanning $\mathbf{P}^{n}$ the weaker version is equivalent to the strong one.

First we observe the following facts.

Remark 2.7. Let $X$ be a set of $n+3$ distinct points of $\mathbf{P}^{n}$ in $G L$ position. This means that $n$ points of $X$ are never on a linear subspace $\mathbf{P}^{n-2}$, and $n+2$ points are never on a linear subspace $\mathbf{P}^{n-1}$. After suitable changing of coordinates, we may assume $X=\left\{P_{0}, \ldots, P_{n}, Q_{1}, Q_{2}\right\}$ where $P_{0}, \ldots, P_{n}$ are the coordinate points and $Q_{1}:=\left(y_{0}, \ldots, y_{n}\right), Q_{2}:=\left(z_{0}, \ldots, z_{n}\right)$.

Let $D_{i j}$ be the minor $\left|\begin{array}{l}y_{i} y_{j} \\ z_{i} z_{j}\end{array}\right|$. Then

(i) If for some $i$ we have $y_{i}=0$ then $y_{j} \neq 0$ for $j \neq i$.

(ii) If $y_{i}=0$ for some $i=0, \ldots, n$, then $z_{i} \neq 0$.

(iii) If for some integers $i, j, 0 \leq i<j \leq n$, we have $D_{i j}=0$, then $y_{i} z_{i} y_{j} z_{j} \neq 0$ and $D_{i k} \neq 0$ for every $k \neq i, j$. 
In fact if $y_{i}=y_{j}=0$ with $i \neq j$, then $X_{i}=X_{j}=0$ is a $\mathbf{P}^{n-2}$ containing $n$ points of $X$. If $y_{i}=z_{i}=0$, then $X_{i}=0$ is a $\mathbf{P}^{n-1}$ containing $n+2$ points of $X$. Finally if $D_{i j}=0$, then $y_{i} z_{j}=y_{j} z_{i}$, hence, using (i) and (ii), we get $y_{i} z_{i} y_{j} z_{j} \neq 0$. On the other hand if $D_{i j}=D_{i k}=0$, it is easy to see that we have again $n$ points of $X$ on a $\mathbf{P}^{n-2}$. This proves the remark.

Proposition 2.8. If $X$ consists of six points in $\mathbf{P}^{3}$ or seven points in $\mathbf{P}^{4}$, the following conditions are equivalent:

(a) The points of $X$ are in GL position.

(b) The points of $X$ are in $\mathrm{gl}$ position.

Proof. By Proposition 2.6, we must prove (a) $\Rightarrow$ (b). In both cases we have $n+3$ points in $\mathbf{P}^{n}$; as above we may assume $X=\left\{P_{0}, \ldots, P_{n}, Q_{1}, Q_{2}\right\}$ where $P_{0}, \ldots, P_{n}$ are the coordinate points and $Q_{1}:=\left(y_{0}, \ldots, y_{n}\right), Q_{2}:=$ $\left(z_{0}, \ldots, z_{n}\right)$.

Let $n=3$; by Remark 2.7 we can choose a permutation $\{i, j, h, k\}$ of $\{0, \ldots, 3\}$ such that $y_{i}, y_{j}, z_{h}, z_{k}, D_{i j}, D_{h k}$ are different from zero. Then it is easy to see that the hyperplane spanned by $P_{h}, P_{k}$ and $Q_{1}$ does not contain any other points of $X$ and however we choose four points of $X$ containing $P_{i}, P_{j}, Q_{2}$ they are not on a hyperplane.

Let now $n=4$; in the same way as before, using Remark 2.7, we may assume that $y_{i}, y_{j}, z_{h}, z_{k}, z_{r}, D_{i j}, D_{h k}, D_{h r}, D_{k r}$ are different from zero where $\{i, j, h, k, r\}$ is a permutation of $\{0, \ldots, 4\}$.

Then it is easy to see that the hyperplane spanned by $P_{h}, P_{k}, P_{r}$ and $Q_{1}$ does not contain other points of $X$ and however we choose 5 points of $X$ containing $P_{i}, P_{j}, Q_{2}$ they are not on a hyperplane.

We end this section by relating our Theorem 3.5 with a similar and recent result by Einsenbud and Koh. They extended the theorem by Green and Lazarsfeld by proving that a set of $\left(\begin{array}{c}n+t-1 \\ n\end{array}\right)+n-i$ points in $\mathbf{P}^{n} \quad(1 \leq i \leq n)$ imposing independent conditions on forms of degree $t-1$, verifies property $\left(N_{i}\right)$. We remark that, for this number of points, imposing independent conditions on forms of degree $t-1$, is equivalent to saying that every subset of $\left(\begin{array}{c}n+t-1 \\ n\end{array}\right)$ points is not on an hypersurface of degree $t-1$. Hence, if $t=2$, this condition is equivalent to general position. We guess that it should imply our $g l$ position, but, for the moment, we cannot prove it. However the following example shows that also if $t \geq 2$, our result is not a consequence of Einsenbud and Koh theorem.

Let $X=\left\{P_{0}, \ldots, P_{3}, Q_{1}, \ldots, Q_{8}\right\} \subseteq \mathbf{P}^{3}$ where $P_{0}, \ldots, P_{3}$ are the coordinate points and $Q_{1}=(0,1,0,1), Q_{2}=(1,0,1,0), Q_{3}=(1,1,0,0)$, $Q_{4}=(0,0,1,1), Q_{5}=(0,1,1,0), Q_{6}=(1,0,0,1), Q_{7}=(1,1,1,1)$, $Q_{8}=(0,1,1,2)$.

It is easy to see that these points are in $g l$ position by looking at the plane spanned by $P_{1}, Q_{2}, Q_{4}$. On the other hand, if we delete $P_{0}$ and $P_{3}$, we get ten points which lie on a quadric. Hence these points do not impose independent conditions on forms of degree two.

\section{FURTHER APPLICATIONS}

As a first application of the previous results we give a complete description of the minimal free resolution of points in generic position lying on a rational normal curve in $\mathbf{P}^{n}$. 
Let $C$ be the rational normal curve in $\mathbf{P}^{n}$. It is known that $C$ is the image of the morphism $\varphi: \mathbf{P}^{1} \rightarrow \mathbf{P}^{n}$ defined by $\varphi(t, u)=\left(t^{n}, t^{n-1} u, \ldots, t u^{n-1}, u^{n}\right)$. Hence the homogeneous coordinate ring of $C$ is the ring

$$
A=k\left[t^{n}, t^{n-1} u, \ldots, t u^{n-1}, u^{n}\right] \text {. }
$$

It follows that the Hilbert function of $C$ is $H_{C}(r)=n r+1$ for every $r \geq 0$. Since $A$ is Cohen-Macaulay of initial degree two and socle degree one we get that $A$ is a short graded algebra with a linear resolution:

$$
0 \rightarrow R(-n)^{a_{n-1}} \rightarrow \cdots \rightarrow R(-2)^{a_{1}} \rightarrow R \rightarrow A \rightarrow 0
$$

where $a_{j}:=a_{j}(C)=j\left(\begin{array}{c}n \\ j+1\end{array}\right)$ for every $j=1, \ldots, n-1$.

If we consider a set $X$ of $s$ points in generic position lying on $C$, then they lie on a quadric and thus we must have $s=H_{X}(2) \leq H_{C}(2)=2 n+1$. On the other hand if we have $n+1 \leq s \leq 2 n+1$ points lying on $C$, they are in general position and so in generic position.

Proposition 3.1. Let $X$ be a set of $s=2 n+1-i, 0 \leq i \leq n$, points lying on the rational normal curve $C$ in $\mathbf{P}^{n}$. Then

$$
a_{j}(X)= \begin{cases}a_{j}(C) & \text { if } j \geq i+1, \\
j\left(\begin{array}{c}
n+2 \\
j+1
\end{array}\right)-s\left(\begin{array}{c}
n \\
j-1
\end{array}\right) & \text { if } 1 \leq j \leq i .\end{cases}
$$

Proof. The points of $X$ are in general position hence, by the result of Green and Lazarsfeld, $b_{j}=0$ for every $j \leq i$. It follows by Proposition 1.6 that $a_{j}(X)=j\left(\begin{array}{c}n+2 \\ j+1\end{array}\right)-s\left(\begin{array}{c}n \\ j-1\end{array}\right)$ for every $j \leq i+1$.

Let now $j \geq i+1$; then $2 n+1-i \geq 2 n-j+2$. Let $Y$ be any subset of $X$ consisting of $2 n-j+2$ points. By Corollary 1.4, we get $a_{j}(C) \leq a_{j}(X) \leq a_{j}(Y)$. Since the points of $Y$ are again in general position and $2 n-j+2=2 n+1-$ $(j-1)$, by the result of Green and Lazarsfeld we get $b_{j-1}(Y)=0$ hence, by Proposition 1.6, $a_{j}(Y)=j\left(\begin{array}{c}n+2 \\ j+1\end{array}\right)-(2 n-j+2)\left(\begin{array}{c}n \\ j-1\end{array}\right)$.

The conclusion follows since it is easy to prove that

$$
j\left(\begin{array}{l}
n+2 \\
j+1
\end{array}\right)-(2 n-j+2)\left(\begin{array}{c}
n \\
j-1
\end{array}\right)= \begin{cases}0 & \text { if } j=n \\
j\left(\begin{array}{c}
n \\
j+1
\end{array}\right) & \text { if } 1 \leq j \leq n-1 .\end{cases}
$$

We remark that the others invariants of the minimal resolution of the points of $X$ can be computed by using Proposition 1.6.

The following example shows that the inequality of Proposition 1.5 is sharp.

Example 3. Let $X$ be a set of $s \leq 2 n+1$ points on the rational normal curve $C$ in $\mathbf{P}^{n}$ and $Y$ any subset of $X$ consisting of $s-1$ points. Let $s=2 n-j+2$, $1 \leq j \leq n$; then by Proposition 3.1 we have $a_{j}(X)=a_{j}(C)=j\left(\begin{array}{c}n \\ j+1\end{array}\right)$ and $a_{j}(Y)=j\left(\begin{array}{c}n+2 \\ j+1\end{array}\right)-(2 n-j+1)\left(\begin{array}{c}n \\ j-1\end{array}\right)$. As in the proof of Proposition 3.1 we see that $j\left(\begin{array}{c}n+2 \\ j+1\end{array}\right)-(2 n-j+2)\left(\begin{array}{c}n \\ j-1\end{array}\right)=j\left(\begin{array}{c}n \\ j+1\end{array}\right)$ and then $a_{j}(Y)=a_{j}(X)+\left(\begin{array}{c}n \\ j-1\end{array}\right)$ for $1 \leq j \leq n$.

If we have a set $X$ of $s<\left(\begin{array}{c}n+2 \\ 2\end{array}\right)$ points lying on a rational normal curve $C$ in $\mathbf{P}^{n}$, then $a_{n-1} \neq 0$. In fact, by Corollary 1.4 , we get $a_{n-1}(X) \geq a_{n-1}(C)$.

From this it follows that, if we have $n+3<s \leq 2 n+1$ points on the rational normal curve in $\mathbf{P}^{n}$, then they do not satisfy the (MRC). In these cases we 
have $m \leq n-2$, hence to satisfy the (MRC), we need $a_{m+1}=0$. This implies $a_{j}=0$ for every $j \geq m+1$ and in particular $a_{n-1}=0$.

Lorenzini proved this fact for seven points in $\mathbf{P}^{3}$ and for 8 points in $\mathbf{P}^{4}$ lying on the corresponding rational normal curve (see [L1]).

In the following proposition we show that for seven points in $\mathbf{P}^{3}$ in general position also the converse holds.

Proposition 3.3. Let $X$ be a set consisting of seven points in $\mathbf{P}^{3}$ in general position.

Then $X$ satisfies the (MRC) if and only if the points do not lie on a rational normal curve in $\mathbf{P}^{3}$.

Proof. Since the points are in general position, they are in generic position. In this case $m=1$ and then $X$ satisfies the (MRC) if and only if $a_{2}=0$. We only need to prove that if $a_{2} \neq 0$ the points lie on a rational normal curve. If $a_{2} \neq 0$, we have a nonzero element $\alpha=\sum_{i=0}^{3} e_{i} \otimes F_{i} \in\left[\Lambda V \otimes I_{2}\right] \cap K_{1}$, where $I$ is the defining ideal of $X$ in $k\left[X_{0}, \ldots, X_{3}\right]$. In particular $F_{i} \in I_{2}$ for every $i=0, \ldots, 3$, and $\sum_{i=0}^{3} X_{i} F_{i}=0$. Since the points are in generic position, $\operatorname{dim}_{k} I_{2}=3$ and so we may write, for example, $F_{3}=\sum_{i=0}^{2} \alpha_{i} F_{i}$. Hence $\sum_{i=0}^{2} X_{i} F_{i}+X_{3}\left(\sum_{i=0}^{2} \alpha_{i} F_{i}\right)=\sum_{i=0}^{2}\left(X_{i}+\alpha_{i} X_{3}\right) F_{i}=0$.

Now $m_{0}:=X_{0}+\alpha_{0} X_{3}, m_{1}:=X_{1}+\alpha_{1} X_{3}$, and $m_{2}:=X_{2}+\alpha_{2} X_{3}$, form a regular sequence in $R$, hence we have $F_{0}=l_{2} m_{1}-l_{1} m_{2}, F_{1}=-l_{2} m_{0}+l_{0} m_{2}$, $F_{2}=l_{1} m_{0}-l_{0} m_{1}$, for some $l_{0}, l_{1}, l_{2} \in R_{1}$. It follows that our points lie on the locus

$$
C:\left\{\operatorname{rank}\left(\begin{array}{ccc}
m_{0} & m_{1} & m_{2} \\
l_{0} & l_{1} & l_{2}
\end{array}\right) \leq 1\right\}
$$

In order to prove that $C$ is a rational normal curve, we need only to prove that for any $(t, u) \neq(0,0)$ the linear forms $t m_{0}+u l_{0}, t m_{1}+u l_{1}, t m_{2}+u l_{2}$ are linearly independent (see [H, p. 104]).

To prove this assertion we need firstly to prove that $F_{1}, F_{2}$ are independent. But if

$$
0=\lambda F_{1}+\mu F_{2}=\lambda\left(l_{0} m_{2}-l_{2} m_{0}\right)+\mu\left(l_{1} m_{0}-l_{0} m_{1}\right)
$$

for some $\lambda, \mu \in k$, then

$$
m_{0}\left(\mu l_{1}-\lambda l_{2}\right)=l_{0}\left(\mu m_{1}-\lambda m_{2}\right)
$$

Since the points are in general position, the quadric $F_{1}$ is irreducible, hence $m_{0}$ and $l_{0}$ are independent. This implies $\mu m_{1}-\lambda m_{2}=\sigma m_{0}$ for some $\sigma \in k$; since $m_{0}, m_{1}, m_{2}$ are independent, we get $\lambda=\mu=0$ as desired.

Now let assume that for some $\lambda_{0}, \lambda_{1}, \lambda_{2} \in k$ we have $\lambda_{0}\left(t m_{0}+u l_{0}\right)$ $=\lambda_{1}\left(t m_{1}+u l_{1}\right)+\lambda_{2}\left(t m_{2}+u l_{2}\right)$ that is $\lambda_{0}\left(t m_{0}+u l_{0}\right)=t\left(\lambda_{1} m_{1}+\lambda_{2} m_{2}\right)+$ $u\left(\lambda_{1} l_{1}+\lambda_{2} l_{2}\right)$.

If for example $t \neq 0$ we get $\lambda_{1} m_{1}+\lambda_{2} m_{2}=\lambda_{0} m_{0}+\frac{u}{t}\left(\lambda_{0} l_{0}-\lambda_{1} l_{1}-\lambda_{2} l_{2}\right)$. 
This implies

$$
\begin{aligned}
& \operatorname{det}\left(\begin{array}{ll}
m_{0} & \lambda_{1} m_{1}+\lambda_{2} m_{2} \\
l_{0} & \lambda_{1} l_{1}+\lambda_{2} l_{2}
\end{array}\right) \\
& \quad=\lambda_{1} F_{2}-\lambda_{2} F_{1}=m_{0}\left(\lambda_{1} l_{1}+\lambda_{2} l_{2}\right)-l_{0}\left[\lambda_{0} m_{0}+\frac{u}{t}\left(\lambda_{0} l_{0}-\lambda_{1} l_{1}-\lambda_{2} l_{2}\right)\right] \\
& \quad=m_{0}\left(\lambda_{1} l_{1}+\lambda_{2} l_{2}-\lambda_{0} l_{0}\right)+\frac{u}{t} l_{0}\left(\lambda_{1} l_{1}+\lambda_{2} l_{2}-\lambda_{0} l_{0}\right) \\
& \quad=\left(m_{0}+\frac{u}{t} l_{0}\right)\left(\lambda_{1} l_{1}+\lambda_{2} l_{2}-\lambda_{0} l_{0}\right) .
\end{aligned}
$$

Since in $I_{2}$ there is no reducible quadric, we must have $\lambda_{1} F_{2}-\lambda_{2} F_{1}=0$, hence $\lambda_{1}=\lambda_{2}=0$. Thus $\lambda_{0}\left(t m_{0}+u l_{0}\right)=0$ which implies $\lambda_{0}=0$, otherwise $m_{0}=-\frac{u}{t} l_{0}$ and $F_{1}$ would be reducible. Since the same argument works also in the case $u \neq 0$, the conclusion follows.

Now we come to the last application of our results.

Let $X$ be a set of $n+2$ distinct points spanning $\mathbf{P}^{n}$. Then, as usual, we may assume $X=\left\{P_{0}, \ldots, P_{n}, Q\right\}$ where $P_{0}, \ldots, P_{n}$ are the coordinate points. Let $Q:=\left(u_{0}, \ldots, u_{n}\right)$ and let $s$ be the number of nonzero coordinates of $Q$. Then $2 \leq s \leq n+1$ and it is clear that different values of $s$ correspond to different configurations of the points. In a recent paper it has been shown that these different configurations have different free resolutions (see [HSV, Theorem $\mathrm{C}$ ]). We prove now that this is also the case for five points in $\mathbf{P}^{2}$, while for six points in $\mathbf{P}^{3}$ we show that different geometrical configurations can have the same numerical resolution. In fact we can give a complete description of all the possible free resolutions for five points in $\mathbf{P}^{2}$ and six in $\mathbf{P}^{3}$.

Proposition 3.6. Let $X$ be a set of five points spanning $\mathbf{P}^{2}$. The minimal free resolution of the homogeneous coordinate ring $A$ of $X$ is given by

(i) $0 \rightarrow R(-4)^{2} \rightarrow R(-3)^{2} \oplus R(-2) \rightarrow R \rightarrow A \rightarrow 0$ if the points are in generic position.

(ii) $0 \rightarrow R(-5) \oplus R(-3) \rightarrow R(-4) \oplus R(-2)^{2} \rightarrow R \rightarrow A \rightarrow 0$ if the points are not in generic position.

Proof. If the points are in generic position, the Hilbert function of an artinian reduction $B$ of $A$ is given by $H_{B}(0)=1, H_{B}(1)=H_{B}(2)=2$ and $H_{B}(n)=0$ for every $n \geq 3$. From this we get that the defining ideal is generated by a quadric and two cubics. The conclusion follows.

If the points of $X$ are not in generic position then $H_{X}(2)=4, H_{X}(i)=5$ for $i \geq 3$. From this it follows that the Hilbert function of an artinian reduction $B$ of $A$ is given by $H_{B}(1)=2, H_{B}(2)=H_{B}(3)=1$ and $H_{B}(n)=0$ for $i \geq 4$. Hence the resolution of $A$ is given by $0 \rightarrow R(-5) \oplus R(-4)^{b_{2}} \oplus R(-3)^{a_{2}} \rightarrow R(-4)^{c_{1}} \oplus R(-3)^{b_{1}} \oplus R(-2)^{2} \rightarrow R \rightarrow A \rightarrow 0$.

Since $B_{3} \neq 0$ and $\operatorname{dim}_{k} B_{2}=1$, the socle of $B$ has no elements of degree two, hence $b_{2}=0$. This implies $a_{2} \neq 0$ otherwise $B$ would be Gorenstein. But $a_{2}<\operatorname{dim}_{k} B_{1}$, hence $a_{2}=1$. The conclusion follows.

Now let $X$ be a set of six points spanning $\mathbf{P}^{3}$. It is not difficult to prove that they are in generic position if and only if four points of $X$ are never on a line. (see [CRV3] for a more general result). It follows that the geometrical configurations for $X$ are the following. 
$C_{1}$ : The points of $X$ are not in generic position.

$C_{2}$ : The points of $X$ are in $G L$ position, or, which is the same, five points of $X$ are never on a plane and three never on a line.

$C_{3}$ : There are five points of $X$ on a plane but four are never on a line.

$C_{4}$ : Three points of $X$ are on a line and three on another line.

$C_{5}$ : Five points of $X$ are never on a plane, but three are on a line and the other three are not on a line.

Proposition 3.7. Let $X$ be a set of six points spanning $\mathbf{P}^{3}$. The minimal free resolution of the homogeneous coordinate ring $A$ of $X$ is given by

(a) $0 \rightarrow R(-6) \oplus R(-4)^{2} \rightarrow R(-5)^{2} \oplus R(-3)^{6} \rightarrow R(-4) \oplus R(-2)^{5} \rightarrow R \rightarrow$ $A \rightarrow 0$ in case $C_{1}$. $\mathrm{C}_{2}$.

(b) $\quad 0 \rightarrow R(-5)^{2} \rightarrow R(-4)^{3} \oplus R(-3)^{2} \rightarrow R(-2)^{4} \rightarrow R \rightarrow A \rightarrow 0$ in case

(c) $0 \rightarrow R(-5)^{2} \oplus R(-4) \rightarrow R(-4)^{4} \oplus R(-3)^{4} \rightarrow R(-3)^{2} \oplus R(-2)^{4} \rightarrow R \rightarrow$ $A \rightarrow 0$ in case $C_{3}$ or $C_{4}$.

(d) $0 \rightarrow R(-5)^{2} \rightarrow R(-4)^{3} \oplus R(-3)^{3} \rightarrow R(-3) \oplus R(-2)^{4} \rightarrow R \rightarrow A \rightarrow 0$ in case $C_{5}$.

Proof. We prove the result in several steps.

Step 1. If the points are not in generic position the Hilbert function of $A$ is $H_{A}(0)=1, H_{A}(1)=4, H_{A}(2)=5$ and $H_{A}(i)=6$ for $i \geq 3$. From this it follows that the Hilbert function of an artinian reduction $B=S / J$ of $A$ is given by $H_{B}(0)=1, H_{B}(1)=3, H_{B}(2)=H_{B}(3)=1$ and $H_{B}(n)=0$ for every $n \geq 4$. Hence the resolution of $A$ is given by

$$
\begin{aligned}
0 & \rightarrow R(-6) \oplus R(-5)^{b_{3}} \oplus R(-4)^{a_{3}} \rightarrow R(-5)^{c_{2}} \oplus R(-4)^{b_{2}} \oplus R(-3)^{a_{2}} \\
& \rightarrow R(-4)^{c_{1}} \oplus R(-3)^{b_{1}} \oplus R(-2)^{5} \rightarrow R \rightarrow A \rightarrow 0 .
\end{aligned}
$$

Since $\operatorname{dim}_{k} B_{2}=\operatorname{dim}_{k} B_{3}=1$, it is clear that $J$ has five generators in degree two and one in degree four. Hence $b_{1}=0$ and $c_{1}=1$. By the alternating sum we get $a_{2}=6$ and $a_{3}=b_{2}+2$. If $a_{3}=\operatorname{dim}_{k} B_{1}=3, B_{1}$ would be in the socle and $B_{2}=0$. Hence $a_{3}=2$ and $b_{2}=0$. In the same way we get $b_{3}<\operatorname{dim}_{k} B_{2}=1$, that is $b_{3}=0$ and $c_{2}=2$. This proves (a).

Now we may assume that the points are in generic position. Hence the Hilbert function of an artinian reduction $B=S / J$ of $A$ is given by $H_{B}(0)=1$, $H_{B}(1)=3, H_{B}(2)=2$ and $H_{B}(n)=0$ for every $n \geq 3$. This implies that the resolution of $A$ is given by

$0 \rightarrow R(-5)^{2} \oplus R(-4)^{a_{3}} \rightarrow R(-4)^{b_{2}} \oplus R(-3)^{a_{2}} \rightarrow R(-3)^{b_{1}} \oplus R(-2)^{4} \rightarrow R \rightarrow A \rightarrow 0$.

Step 2. If the points of $X$ are in generic position then $a_{3} \leq 1$ and $2 \leq a_{2} \leq 4$.

Proof. If $a_{3} \geq 2$, we would have two linear forms, say $L, M \in S_{1}$ in the socle of $B$, hence $(L, M)_{2} \subseteq J_{2}$. Since $\operatorname{dim}_{k}(L, M)_{2}=5$ and $\operatorname{dim}_{k} J_{2}=4$ we get a contradiction. Moreover by the alternating sum we get $a_{2}=2+b_{1}$. Hence if $a_{2}>4$ then $a_{1}+b_{1}>4+4-2=6$. This is a contradiction by the main result in [ERV].

Since our points span $\mathbf{P}^{3}$, as usual we may assume $X=\left\{P_{0}, \ldots, P_{3}, Q_{1}\right.$, $\left.Q_{2}\right\}$ where $P_{0}, \ldots, P_{3}$ are the coordinate points and $Q_{1}:=\left(y_{0}, \ldots, y_{3}\right), Q_{2}$ $:=\left(z_{0}, \ldots, z_{3}\right)$. 
By the theorem of Green and Lazarsfeld if the points are in $G L$ position then property $\left(N_{1}\right)$ holds, hence $b_{1}=0$

Step 3. If three points of $X$ are never on a line, then $a_{3}=0$.

Proof. We have $a_{3}=\operatorname{dim}_{k}\left[\left(\bigwedge^{2} V \otimes I(X)_{2}\right) \cap K_{2}\right]$; if $\alpha \in\left(\bigwedge^{2} V \otimes I(X)_{2}\right) \cap K_{2}$, then using the description given at the beginning of $\S 2$, we may write $\alpha=$ $\sum_{0 \leq i<j \leq 3} \lambda_{i j} \varepsilon_{i j} \otimes X_{i} X_{j}$ where $\varepsilon_{i j}=e_{0} \wedge \cdots \wedge \hat{e}_{i} \wedge \cdots \wedge \hat{e}_{j} \wedge \cdots \wedge e_{3}$ and $\lambda_{i j} X_{i} X_{j} \in$ $I(X)_{2}$.

As in Remark 2.7, if three points are never on a line, $Q_{1}, Q_{2}$ have at most one zero coordinate. Hence $\alpha=\varepsilon_{i j} \otimes \lambda_{i j} X_{i} X_{j}$ and since $\alpha \in K_{2}$ we get $\alpha=0$. From this it is clear that if the points of $X$ are in $G L$ position the minimal free resolution is

$$
0 \rightarrow R(-5)^{2} \rightarrow R(-4)^{3} \oplus R(-3)^{2} \rightarrow R(-2)^{4} \rightarrow R \rightarrow A \rightarrow 0 .
$$

This proves (b).

Step 4. In case $C_{3}$ we have $a_{2}=4$ and $a_{3}=1$.

Proof. By Step 2 we need only to prove that $a_{3} \geq 1$ and $a_{2} \geq 4$.

We may suppose that five points are on $X_{0}=0$, that is $y_{0}=z_{0}=0$. It is clear that we have $\delta_{3}\left(e_{1} \wedge e_{2} \wedge e_{3} \otimes X_{0}\right) \in \stackrel{2}{\Lambda} V \otimes I_{2} \cap K_{2}$, hence $a_{3} \geq 1$.

On the other hand by Proposition 2.1 we have $a_{2}+\operatorname{rank}\left(M_{1}\right)=8$. Now looking inside the matrix $M_{1}$ it is easy to see that $\operatorname{rank} M_{1} \leq 4$ and hence $a_{2} \geq 4$.

Step 5. In case $C_{4}$ we have $a_{2}=4$ and $a_{3}=1$.

Proof. It is clear that we may assume $r: X_{0}=X_{1}=0, s: X_{2}=X_{3}=0$, $y_{0}=y_{1}=z_{2}=z_{3}=0$ and $y_{2} y_{3} z_{0} z_{1} \neq 0$. It is clear that

$$
\delta_{3}\left(e_{1} \wedge e_{2} \wedge e_{3} \otimes X_{0}-e_{0} \wedge e_{2} \wedge e_{3} \otimes X_{1}\right) \in \bigwedge^{2} V \otimes I_{2} \cap K_{2},
$$

hence $a_{3} \geq 1$. Also it is easy to see that the matrix $M_{1}$ has only four rows which are different from zero, hence $\operatorname{rank}\left(M_{1}\right) \leq 4$ and $a_{2} \geq 4$. Steps 4 and 5 prove (c).

Step 6. In case $C_{5}$ we have $a_{2}=3$ and $a_{3}=0$.

Proof. Let us first consider the case where there is a line containing two coordinate points and one "extra" point. We may assume that $P_{2}, P_{3}, Q_{1}$ are on $X_{0}=X_{1}=0$. Then $y_{2} y_{3} \neq 0$ and since five points are never on a plane also $z_{0} z_{1} \neq 0$; moreover $\left(z_{2}, z_{3}\right) \neq(0,0)$ otherwise $P_{0}, P_{1}, Q_{2}$ would lie on $X_{2}=X_{3}=0$. Hence we may assume $y_{0}=y_{1}=0$ and $y_{2} y_{3} z_{0} z_{1} z_{3} \neq 0$. With these assumptions it is easy to see that $a_{3}=0$ and $\operatorname{rank}\left(M_{1}\right)=5$, hence $a_{2}=3$ as desired.

Finally let us assume that there is a line containing $Q_{1}, Q_{2}$ and for example $P_{3}$. With the notations as in Remark 2.7, this means that $D_{01}=D_{02}=D_{12}=0$.

In this case it is easy to see that $y_{0}, y_{1}, y_{2}, z_{0}, z_{1}, z_{2}, D_{23}$ are different from zero, otherwise we get 5 points on a plane.

Hence we may assume for example $z_{3} \neq 0$. Since all the coordinates of $Q_{2}$ are different from 0 we get $a_{3}=0$, while looking inside the matrix $M_{1}$, we can easily prove that $\operatorname{rank}\left(M_{1}\right)=5$. Hence $a_{2}=3$ and the conclusion follows. 
Some of the results here were discovered or confirmed with the help of the computer algebra program COCOA written by A. Giovini and G. Niesi (see [GN]).

\section{REFERENCES}

[BG] E. Ballico and A. V. Geramita, The minimal free resolution of the ideal of $s$ general points in $\mathbf{P}^{3}$, Canad. Math. Soc. Conf. Proc., vol. 6, Amer. Math. Soc., Providence, R.I., 1986, pp. 1-9.

[CRV1] M. P. Cavaliere, M. E. Rossi and G. Valla, On short graded algebras, Commutative Algebra, Salvador, vol. 1430, Lecture Notes in Math., Springer, 1990, pp. 21-31.

[CRV2] _ On the resolution of points in generic position, Comm. Algebra (4) 19 (1991), 1083-1097.

[CRV3] _ On Green-Lazarsfeld and minimal resolution conjecture for $n+3$ points in $\mathbf{P}^{n}, \mathbf{J}$. Pure Appl. Algebra (to appear).

[ERV] J. Elias, R. Robbiano and G. Valla, Number of generators of ideals, Nagoya Math J. 123 (1991), 39-76.

[EK] D. Eisenbud and J. H. Koh, Remarks on points in a projective space, Commutative Algebra, Proceedings of a microprogram held June 15-July 2, 1987, vol. 15, Math. Sci. Research Inst. Publ., Springer, 1989, pp. 157-172.

[EK1] - Some linear syzygies conjectures, Adv. Math. (to appear).

[G] M. Green, Koszul homology and the geometry of projective varieties, J. Differential Geometry 19 (1984), 125-171.

[GL] M. Green and R. Lazarsfeld, Some results on the syzygies of finite sets and algebraic curves, Comput. Math. 67 (1988), 301-314.

[GN] A. Giovini and G. Niesi, CoCoA: A user-friendly system for commutative algebra, design and implementation of symbolic computation systems, 429 , Lecture Notes in Comput. Sci., Springer-Verlag, 1990, pp. 20-30.

[GO] A. V. Geramita and F. Orecchia, On the Cohen-Macaulay type of s lines in $\mathbf{A}^{n+1}$, J. Algebra 70 (1981), 116-140.

[HSV] L. T. Hoa, J. Stükrad and W. Vogel, Toward a structure theory for projective varieties of degree $=$ codimension +2 , Preprint 1989.

[H] J. Harris, Curves in projective space, Séminaire de Mathématiques Supérieures, Université de Montréal, 1982.

[L1] A. Lorenzini, On the Betti numbers of points in the projective space, Thesis, Queen's University, Kingston, Ontario, 1987.

[L2] _ Betti numbers of perfect homogeneous ideals, J. Pure Appl. Algebra 60 (1989), 273-288.

[L3] - The minimal resolution conjecture, J. Algebra (to appear).

[MNV] P. Maroscia, U. Nagel and W. Vogel, On degree bound for the syzygies of a finite set of points in $\mathbf{P}^{n}$, Preprint 1990.

[TV] N. V. Trung and G. Valla, The Cohen-Macaulay type of points in generic position, J. Algebra 125 (1989), 110-119.

Dipartimento di Matematica, Università di Genova, Via L. B. Alberti 4, 16132 Genova, ITALY

E-mail address: valla@igecuniv.bitnet 